\title{
Influence of tensile stress on permeability properties of type 304 stainless steel
}

\author{
$\operatorname{AUTHOR}(S)$ :
}

Kinoshita, K.

\section{CITATION:}

Kinoshita, K.. Influence of tensile stress on permeability properties of type 304 stainless steel. Journal of Applied Physics 2015, 117(17): $17 \mathrm{~B} 713$.

\section{ISSUE DATE:}

2015-05-07

\section{URL:}

http://hdl.handle.net/2433/197279

\section{RIGHT:}

(c) 2015 American Institute of Physics. This article may be downloaded for personal use only. Any other use requires prior permission of the author and the American Institute of Physics. 


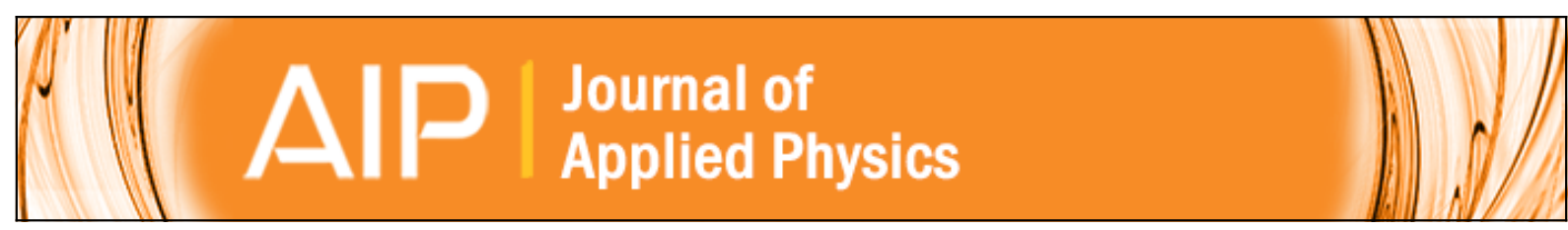

\section{Influence of tensile stress on permeability properties of type 304 stainless steel}

\section{K. Kinoshita}

Citation: Journal of Applied Physics 117, 17 B713 (2015); doi: 10.1063/1.4913819

View online: http://dx.doi.org/10.1063/1.4913819

View Table of Contents: http://scitation.aip.org/content/aip/journal/jap/117/17?ver=pdfcov

Published by the AIP Publishing

\section{Articles you may be interested in}

Nondestructive evaluation of residual stresses in case hardened steels by magnetic anisotropy measurements AIP Conf. Proc. 1430, 1445 (2012); 10.1063/1.4716386

Physical aging of plastoferrites under tensile stress and its effect on microwave properties

J. Appl. Phys. 104, 064108 (2008); 10.1063/1.2978223

Dynamic magnetomechanical properties of Terfenol-D/epoxy pseudo 1-3 composites

J. Appl. Phys. 97, $10 \mathrm{M} 308$ (2005); 10.1063/1.1851889

Dependence of power losses on tensile stress for Fe-Si nonoriented steel up to destruction

J. Appl. Phys. 91, 7854 (2002); 10.1063/1.1446117

Experimental study of laser-driven shock waves in stainless steels

J. Appl. Phys. 84, 5985 (1998); 10.1063/1.368894

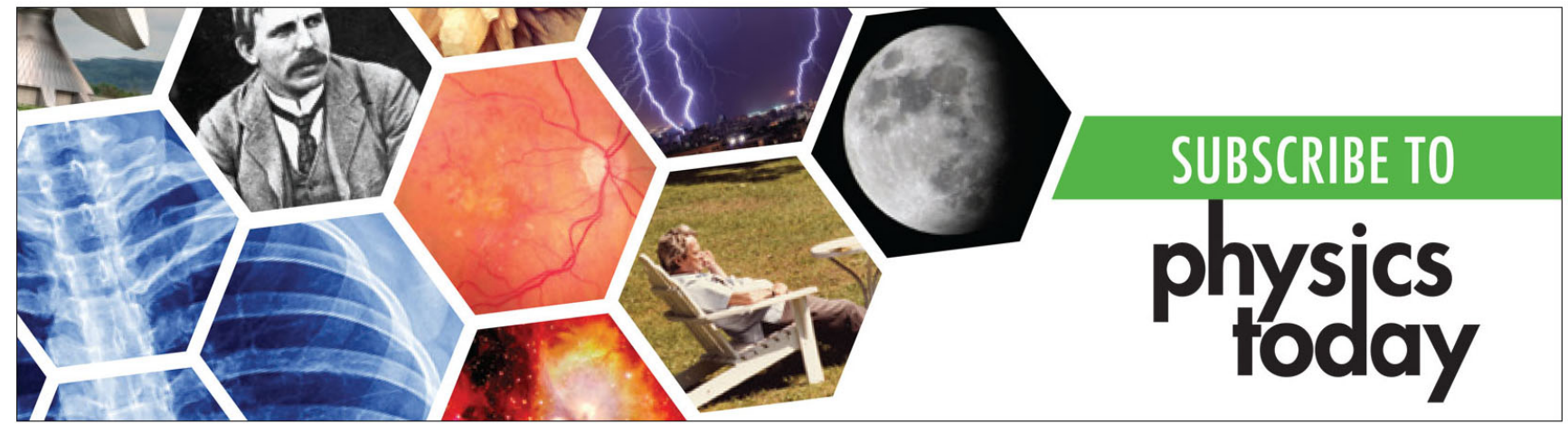




\title{
Influence of tensile stress on permeability properties of type 304 stainless steel
}

\author{
K. Kinoshita ${ }^{\text {a) }}$ \\ Department of Energy Conversion Science, Graduate School of Energy Science, Kyoto University, \\ Yoshida-honmachi, Sakyo-ku, Kyoto 606-8501, Japan
}

(Presented 6 November 2014; received 22 September 2014; accepted 27 October 2014; published online 4 March 2015)

\begin{abstract}
The permeability properties of type SUS304 stainless steel (SUS304 steel) were evaluated under different values of tensile stress using the electromagnetic impedance method. The impedance-magnetic-field curve of SUS304 steel, which corresponds to the permeability-magnetic field-curve, was measured under tensile stresses of 0,70 , and $140 \mathrm{MPa}$ for specimens subjected to prestrains of 5\% to $40 \%$ to change the martensite fraction. The impedance curves were measured in the length (tensile) direction and the width direction. The results showed that the tensile direction was the magnetic hard axis of the martensite phase in SUS304 steel. The applied stress sensitivity of the permeability in SUS304 steel was affected by the volume fraction, residual stress, stress distribution according to the orientation angle of the martensite phase, and their interactions. (C) 2015 AIP Publishing LLC. [http://dx.doi.org/10.1063/1.4913819]
\end{abstract}

\section{INTRODUCTION}

The ferromagnetic martensite phase (m-phase) of austenitic SUS304 steel is induced by plastic deformation. We have previously proposed using this $\mathrm{m}$-phase formed during production and machining as an indicator of deterioration and have investigated the magnetic properties of the phase. ${ }^{1,2}$ The magnetic properties were confirmed to strongly depend on the volume fraction, aspect ratio, and orientation angle of the m-phase, as well as on the internal stress. However, the effect of internal stress on the magnetic properties has only been evaluated before and after annealing, ${ }^{1}$ without a detailed investigation. Although it has been previously reported $^{3}$ that the internal stress has a possible effect on the magnetic properties of the m-phase, a detailed survey has been not conducted. Moreover, it is difficult to accurately induce internal stress in a specimen.

In this study, the permeability properties of SUS304 steel were measured under different tensile stress values using an electromagnetic impedance (EMI) method, ${ }^{1,2}$ and the dependence of the permeability properties of the mphase on the applied stress was evaluated. The permeability properties were measured along the length and width for specimens subjected to prestrains of $5 \%$ to $40 \%$ to assess the relationship between the martensite structure and applied stress.

\section{THEORY}

The shape, distribution mode, and orientation distribution of the m-phase generated in SUS304 steel by plastic deformation are dependent on the mechanical test conditions. $^{2,4,5}$ In the case of plain tensile deformation, ${ }^{2}$ needle-like m-phase particles are orientated at nearly $45^{\circ}$ to the load direction at a prestrain of $5 \%$, and the orientated angle decreases with increasing prestrain. Therefore, because the m-phase stress generated by applied stress changes with the amount of prestrain, it is important to understand the relationship between the internal stress, orientation angle, and permeability of the $\mathrm{m}$-phase. Figure 1 shows a schematic illustration of SUS304 steel when the needle-like m-phase particle is modeled as a single, two-dimensional, ellipsoidal inhomogeneity for simplicity. The $x_{1}$ and $x_{2}$ axes are along the directions of the specimen's length and width, respectively. The $x_{1}{ }^{\prime}$ and $x_{2}{ }^{\prime}$ axes are the m-phase particle's long and short axes directions, and $\theta$ is the orientation angle between the $x_{1}$ and $x_{1}{ }^{\prime}$ axes. When tensile stress $\sigma_{0}$ is applied along the $x_{1}$ axis, the elastic modulus and Poisson's ratio of the austenite phase (a-phase) and m-phase, as well as the aspect ratio of the m-phase, determine the stress condition in the m-phase. ${ }^{6}$ However, because the mechanical properties of the m-phase have not been measured yet, the same mechanical properties are considered for both the a-phase and m-phase in many cases. ${ }^{7}$ As a result, the stress of the

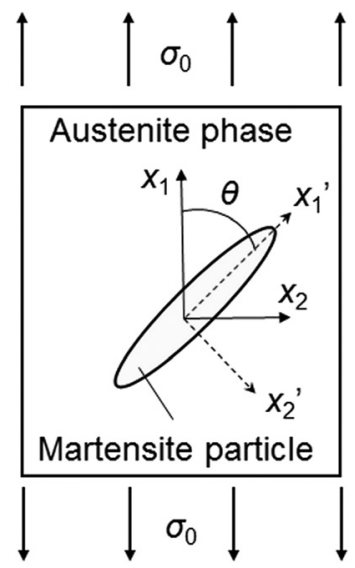

FIG. 1. Schematic of martensite particle model under tensile stress. 
m-phase in the $x_{1}{ }^{\prime}$ - and $x_{2}{ }^{\prime}$-directions is determined by $\sigma_{0}$ and $\theta$, as shown in Eq. $(1)^{8}$

$$
\sigma_{1}{ }^{\prime}=\sigma_{0} \cos ^{2} \theta, \quad \sigma_{2}{ }^{\prime}=\sigma_{0} \sin ^{2} \theta
$$

In this study, because specimens without heat treatment were used, the effect of residual stress generated by the prestrain must be added to Eq. (1). Heat treatment was omitted to investigate the characters of such practical materials because most SUS304 steel, such as the \#400 finish, contains the m-phase formed during production and machining and is used without stress relief annealing after processing. When tensile stress was applied along the $x_{1}$ direction, the amounts of plastic strain in the $x_{1}$-direction in the a-phase and $\mathrm{m}$-phase were different because the proof stress of the m-phase was higher than that of the aphase. The difference in plastic strain thus generated internal tensile stress in the $x_{1}$-direction of the m-phase after unloading. ${ }^{9}$ Without applied stress along the $x_{2}$-direction, the difference in residual strain in the $x_{2}$-direction generated internal compressive stress, which is opposite to the tensile stress along the $x_{1}$-direction. Because elastic stress was applied to the specimens, if the residual stresses in the $x_{1}$ - and $x_{2}$-directions are defined as $\sigma_{1}{ }^{\mathrm{r}}$ and $\sigma_{2}{ }^{\mathrm{r}}$, respectively, the m-phase stresses in the $x_{1}{ }^{\prime}$ - and $x_{2}{ }^{\prime}$-directions can be calculated by superposition, as shown in the following equations:

$$
\begin{aligned}
& \sigma_{1}{ }^{\prime}=\left(\sigma_{0}+\sigma_{1}{ }^{\mathrm{r}}\right) \cos ^{2} \theta+\sigma_{2}{ }^{\mathrm{r}} \sin ^{2} \theta, \\
& \sigma_{2}{ }^{\prime}=\left(\sigma_{0}+{\sigma_{1}}^{\mathrm{r}}\right) \sin ^{2} \theta+\sigma_{2}{ }^{\mathrm{r}} \cos ^{2} \theta .
\end{aligned}
$$

Because plastic deformation satisfies the incompressibility condition, the residual strain in the $x_{2}$-direction is half of that in the $x_{1}$-direction. Therefore, as the tensile stress $\sigma_{1}{ }^{\mathrm{r}}$ is greater than the compressive stress $\sigma_{2}{ }^{\mathrm{r}}, \sigma_{1}{ }^{\prime}$, and $\sigma_{2}{ }^{\prime}$ are components of the tensile stress with the same magnitude at $\theta=45^{\circ}$

We assume that the permeability in an arbitrary direction depends only on the stress in the same direction. The permeabilities in the $x_{1}{ }^{\prime}$ - and $x_{2}{ }^{\prime}$-directions are defined as a function of $\sigma_{1}{ }^{\prime}, \mu_{1}{ }^{\prime}\left(\sigma_{1}{ }^{\prime}\right)$, and a function of $\sigma_{2}{ }^{\prime}, \mu_{2}{ }^{\prime}\left(\sigma_{2}{ }^{\prime}\right)$, respectively, and the permeabilities in the $x_{1}$ - and $x_{2}$-directions are obtained by performing coordinate transformation. The EMI method measures the impedance as a manifestation of the permeability that is changed by a static magnetic field. When a magnetic field is applied in only one direction, which is the same direction in which the permeability is measured, the permeability along the measurement direction is given as follows:

$$
\begin{aligned}
& \mu_{1}=\mu_{1}^{\prime}\left(\sigma_{1}^{\prime}\right) \cos ^{2} \theta+\mu_{2}^{\prime}\left(\sigma_{2}^{\prime}\right) \sin ^{2} \theta \\
& \text { if the magnetic field is in the } x_{1} \text {-direction, } \\
& \mu_{2}=\mu_{1}^{\prime}\left(\sigma_{1}^{\prime}\right) \sin ^{2} \theta+\mu_{2}^{\prime}\left(\sigma_{2}^{\prime}\right) \cos ^{2} \theta \\
& \text { if the magnetic field is in the } x_{2} \text {-direction. }
\end{aligned}
$$

From Eqs. (4) and (5), it is predicted that $\mu_{1}$ and $\mu_{2}$ exhibit similar behavior to $\sigma_{0}$ at $\theta=45^{\circ}$.

\section{EXPERIMENTAL}

The specimens were cold-rolled SUS304 steel plates (2B finish), which had a dumbbell shape with parallel lengths of $146 \mathrm{~mm}$, and a thickness of $3 \mathrm{~mm}$, subjected to prestrains of $5 \%$ to $40 \%$ to change the martensite fraction. Tensile stress was applied by a tensile testing machine, and the stress values were 70 and $140 \mathrm{MPa}$, below the proportional limit of $150 \mathrm{MPa}$. A rectangular coil with dimensions of $6 \times 8 \times 1 \mathrm{~mm}^{3}$ was used for measuring impedance, and the wire had a diameter of $60 \mu \mathrm{m}$. The winding numbers of the coil for the measurements in the length and width directions were 195 and 197 turns, respectively. The impedance was measured using an LCR meter (lift-off distance $=20 \mu \mathrm{m}, \mathrm{AC}$ frequency $=3.5 \mathrm{MHz}$, AC voltage $=0.5 \mathrm{~V}$ ). The test proceeded as follows. A load was applied to a predefined stress value, which was maintained. After demagnetization, the coil impedance was measured only during periods when the magnetic field was maintained using an electromagnet, and the impedance-magnetic-field relation (impedance curve) was obtained. An exact formula cannot be derived for the permeability, because the impedance curve of ferromagnetism is nonlinear. Therefore, the impedance curve was fitted to the exponential function given by Eq. (6), and deterioration such as fatigue was evaluated using the coefficients

$\Delta Z=\left(Z(H)-Z\left(H_{I=0}\right)\right) / Z\left(H_{I=0}\right)=\alpha \exp (-\beta H)+\delta H+\gamma$,

where $\Delta Z$ is the rate of impedance change, $I$ is an excitation current of the electromagnet, and $H$ is the magnetic field. Although $\alpha, \beta, \gamma$, and $\delta$ in Eq. (6) are evaluation parameters, the value of $\phi$, calculated using Eq. (7), was used to evaluate the permeability properties in this study.

$$
\phi=\left|\int_{0}^{200}\{\alpha \exp (-\beta H)+\delta H+\gamma\} d H\right| .
$$

The martensite fraction of the specimens was calculated by assigning the value of $\alpha$ obtained in the experiments to the function of $\alpha$ versus $m$-fraction obtained using results from the literature. ${ }^{3}$

\section{RESULTS AND DISCUSSION}

Figure 2 shows the relationship between $\phi^{\mathrm{L}}$, obtained from the $\Delta Z$ curve in the length direction, and tensile stress. The superscript $\mathrm{L}$ indicates the length direction, and $\bullet$ and $\circ$ represent the result of the two specimens with the same prestrain; the martensite fraction of $\bullet$ is higher than that of $\circ$. For specimens subjected to prestrains other than $10 \%$ and $20 \%, \phi^{\mathrm{L}}$ decreases with increasing tensile stress. Because a decrease in $\phi^{\mathrm{L}}$ indicates a decrease in the variation of the permeability of the m-phase, the direction of the tensile stress is along the magnetic hard axis of the m-phase. Conversely, when the prestrains are $10 \%$ and $20 \%, \phi^{\mathrm{L}}$ does not decrease with increasing tensile stress and change randomly. Table I shows the martensite fraction $V_{\mathrm{f}}$ and $\Delta \phi^{\mathrm{L}}$, which is the average of the difference in $\phi^{\mathrm{L}}$ obtained under tensile stresses of 0 and $140 \mathrm{MPa}$ for specimens subjected to 


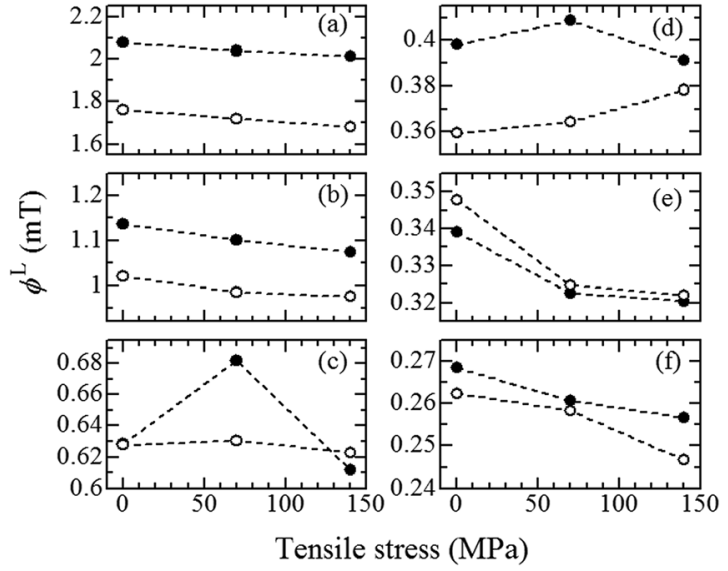

FIG. 2. Relationship between $\phi^{\mathrm{L}}$ and prestrain under different tensile stresses at a prestrain of (a) $40 \%$, (b) $30 \%$, (c) $20 \%$, (d) $10 \%$, (e) $5 \%$, and (f) $0 \%$.

TABLE I. Martensite fraction $V_{\mathrm{f}}$ and $\Delta \phi^{\mathrm{L}}$ which is the average of the difference in $\phi^{\mathrm{L}}$ obtained under tensile stresses of 0 and $140 \mathrm{MPa}$ for specimens subjected to the same prestrain.

\begin{tabular}{llc}
\hline \hline Prestrain $(\%)$ & $V_{\mathrm{f}}(\%)$ & $\Delta \phi^{\mathrm{L}}(\mathrm{mT})$ \\
\hline 40 & 3.8 & 0.068 \\
30 & 1.3 & 0.055 \\
20 & 0.46 & 0.010 \\
10 & 0.19 & 0.0060 \\
5 & 0.17 & 0.022 \\
0 & 0.14 & 0.014 \\
\hline \hline
\end{tabular}

the same prestrain. The values of $\Delta \phi^{\mathrm{L}}$ for specimens subjected to prestrains of $10 \%$ and $20 \%$ are much lower than those of other specimens, which means that their $\Delta Z$ curve is almost unchanged by tensile stress. However, more detailed work is necessary to resolve this issue. Because $\Delta \phi^{\mathrm{L}}$ increases with the martensite fraction $V_{\mathrm{f}}$, a correlation exists between $V_{\mathrm{f}}$ and the stress dependency of the $\Delta Z$ curve.

Figure 3 shows the relationship between $\phi^{\mathrm{T}}$ obtained from the $\Delta Z$ curve in the width direction and the tensile stress; the superscript $\mathrm{T}$ indicates the width direction. $\phi^{\mathrm{T}}$ also decreases with increasing tensile stress, except for prestrains of $10 \%$ and $20 \%$. Therefore, when the m-phase particles are oriented at nearly $45^{\circ}$ to the load direction, the $\mu_{2}$ in the $x_{2}$-direction shows the same dependence on tensile stress as the $\mu_{1}$ in the $x_{1}$-direction, as predicted by Eqs. (4) and (5). Figure 4 shows the relationship between both $\Delta \phi^{\mathrm{L}}$ and $\Delta \phi^{\mathrm{T}}$ and the prestrain. $\Delta \phi^{\mathrm{L}}$ increases almost linearly with increasing prestrain, except for prestrains of $10 \%$ and $20 \%$. However, $\Delta \phi^{\mathrm{T}}$ of the specimen prestrained at $5 \%$ is somewhat lower than that of the specimen prestrained $0 \%$, and the relationship between $\Delta \phi^{\mathrm{T}}$ and prestrains of $0 \%$, $30 \%$, and $40 \%$ is not linear. As described in Sec. II, the orientation angle of the $\mathrm{m}$-phase particles to the load direction changes from $45^{\circ}$ to $0^{\circ}$ with increasing prestrain. Because the martensite fraction in the specimen subjected to a prestrain of 5\% is almost unchanged compared with that in the specimen subjected to a prestrain of $0 \%$ (Table I), the change in $\Delta \phi^{\mathrm{T}}$ is dependent on $\theta, \sigma_{1}{ }^{\mathrm{r}}$, and $\sigma_{2}{ }^{\mathrm{r}}$. Figure 5 shows the relationship between $\sigma_{2}{ }^{\prime}$ and $\theta$ under various values of

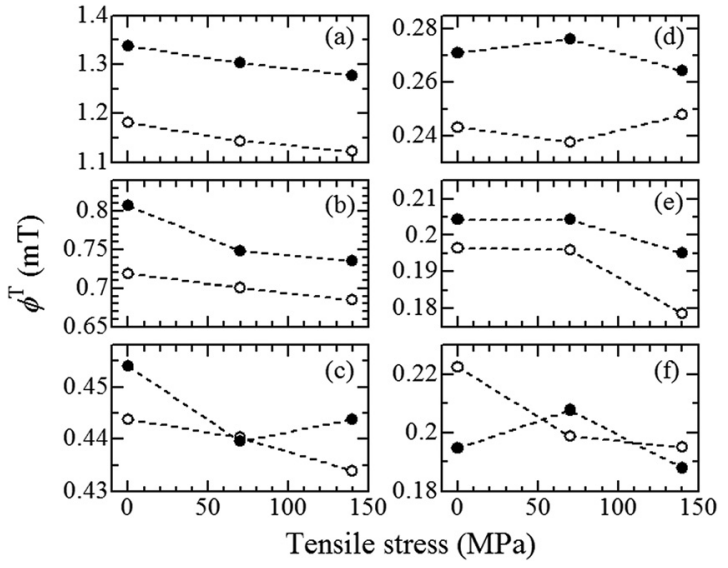

FIG. 3. Relationship between $\phi^{\mathrm{T}}$ and prestrain under different tensile stresses at a prestrain of (a) $40 \%$, (b) $30 \%$, (c) $20 \%$, (d) $10 \%$, (e) $5 \%$, and (f) $0 \%$.

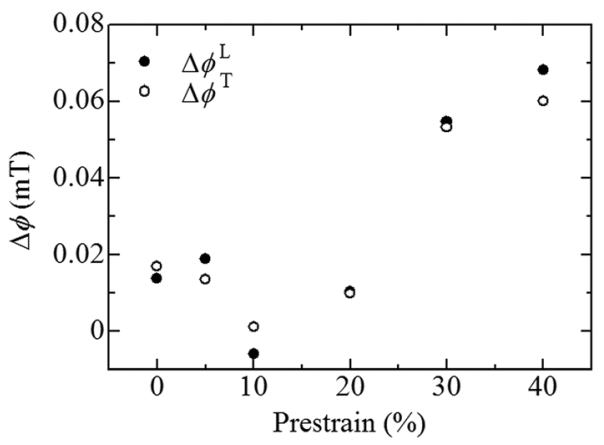

FIG. 4. Relationships between both $\Delta \phi^{\mathrm{L}}$ and $\Delta \phi^{\mathrm{T}}$ and the prestrain.

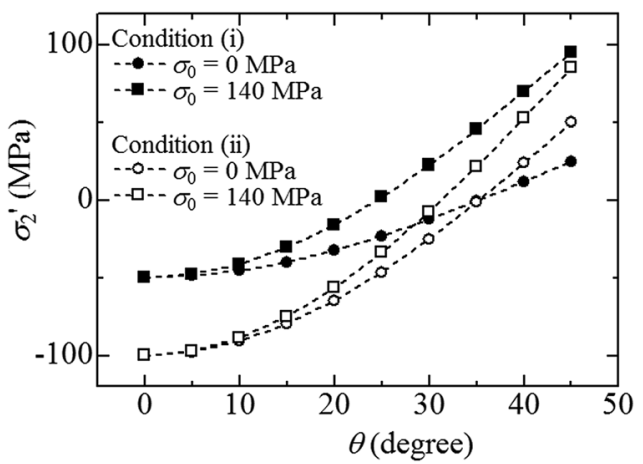

FIG. 5. Relationships between stress in the $x_{2}{ }^{\prime}$-direction and orientation angle under different tensile stresses represented by conditions (i) and (ii).

tensile stress using Eq. (3), when conditions (i) $\sigma_{1}{ }^{\mathrm{r}}=100 \mathrm{MPa}, \quad \sigma_{2}{ }^{\mathrm{r}}=-50 \mathrm{MPa}$, or (ii) $\sigma_{1}{ }^{\mathrm{r}}=200 \mathrm{MPa}$, $\sigma_{2}{ }^{\mathrm{r}}=-100 \mathrm{MPa}$ are assumed. The value of $\sigma_{2}{ }^{\prime}$ decreases with decreasing $\theta$ under all conditions, and $\sigma_{2}{ }^{\prime}$ decreases more under condition (ii) than under condition (i). The difference between values of $\sigma_{2}{ }^{\prime}$ corresponding to $\sigma_{0}=0$ and $140 \mathrm{MPa}$ is smaller under condition (ii) than under condition (i). Therefore, because $\theta$ at a prestrain of $5 \%$ is smaller than that at a prestrain of $0 \%$, and because $\sigma_{1}{ }^{\mathrm{r}}$ and $\sigma_{2}{ }^{\mathrm{r}}$ at a prestrain of $5 \%$ are larger than the corresponding values at a prestrain of $0 \%, \Delta \phi^{\mathrm{T}}$ at a prestrain of $5 \%$ is somewhat lower than $\Delta \phi^{\mathrm{T}}$ at a prestrain of $0 \%$. Further, because the values of 
$\Delta \phi^{\mathrm{T}}$ at prestrains of $30 \%$ and $40 \%$ are also dependent on the martensite fraction, the non-linearity of $\Delta \phi^{\mathrm{T}}$ cannot be explained by the above scenario alone. However, to explain the large variation in the value $\Delta \phi^{\mathrm{T}}$ at a prestrain of $30 \%$, more experiments are necessary.

\section{CONCLUSION}

In summary, it is shown that the tensile-stress direction is the magnetic hard axis of the martensite phase in SUS304 stainless steel. However, the tensile stress dependency of the permeability was not observed at specific prestrains $(10 \%$ and $20 \%$ ). When the martensite phase particles are oriented at nearly $45^{\circ}$ to the load direction, the permeability in the width direction shows the same dependence on tensile stress as the one in the length direction, as predicted theoretically. The sensitivity of the permeability in prestrained SUS 304 stainless steel to applied stress is affected by the volume fraction, residual stress, stress distribution according to the orientation angle in the martensite phase, and their interactions. When the prestrain is less than $5 \%$ and the volume fraction hardly increases, the variation of stress distribution according to the orientation angle in the martensite phase is clear.

\section{ACKNOWLEDGMENTS}

This work was supported by Grant-in-Aid for Scientific Research (C) (No. 23560503).

${ }^{1}$ K. Kinoshita et al., Int. J. Appl. Electromagn. Mech. 39, 375 (2012).

${ }^{2}$ K. Kinoshita et al., Int. J. Appl. Electromagn. Mech. 45, 45 (2014).

${ }^{3}$ K. Mumtaz et al., J. Mater. Sci. 39, 85 (2004).

${ }^{4}$ T. Iwamoto et al., Int. J. Mech. Sci. 40, 173 (1998).

${ }^{5}$ Y. Uematsu et al., J. Soc. Mater. Sci., Jpn. 62, 744 (2013).

${ }^{6}$ T. Mura, Micromechanics of Defects in Solids, 2nd revised ed. (Kluwer Academic, Dordrecht, 1987).

${ }^{7}$ N. Tsuchida et al., J. Jpn. Inst. Met. 72, 769 (2008) (in Japanese).

${ }^{8}$ J. M. Gere and S. P. Timoshenko, Mechanics of Materials, 4th SI ed. (Stanley Thornes, Delta Place, 1999), p. 477.

${ }^{9}$ A. Takimoto et al., Trans. JSME 55, 1909 (1989) (in Japanese). 